\title{
LEFT SEPARATED SPACES WITH POINT-COUNTABLE BASES
}

\author{
BY
}

\author{
WILLIAM G. FLEISSNER ${ }^{1}$
}

\begin{abstract}
Theorem 2.2 lists properties equivalent to left separated spaces in the class of $T_{1}$ with point-countable bases, with examples preventing plausible additions to this list. For example, $X$ is left iff $X$ is $\sigma$-weakly separated or $X$ has a closure preserving cover by countable closed sets, but $X$ is left separated does not imply that $X$ is $\sigma$-discrete. Theorem 2.2 is used to show that the following reflection property holds after properly collapsing a supercompact cardinal to $\omega_{2}$ : If $X$ is a not $\sigma$-discrete metric space, then $X$ has a not $\sigma$ discrete subspace of cardinality less than $\omega_{2}$. Similar reflection properties are shown true in some models and false in others.
\end{abstract}

1. Introduction. If $P$ is a property of topological spaces, let $\mathbf{S}(\mathbf{P})$ denote the assertion: "whenever $X$ is a space with property $P$, then $X$ has a subspace of cardinality $\leq \omega_{1}$ with property $P$ ". In particular, we consider $\mathbf{S}_{\mathbf{1}} \equiv S$ (metric, not $\sigma$-discrete). The proof of the consistency of $S_{1}$ leads to consideration of $\mathbf{S}_{2} \equiv S$ $\left(T_{1}\right.$, point-countable base, not left separated) and $\mathbf{S}_{3} \equiv S\left(T_{1}\right.$, point-countable base, not $\sigma$-relatively discrete).

$\S 2$ starts with an explanation of the topological terminology used in Theorem 2.2 , which lists properties equivalent to left separated in the class of $T_{1}$ spaces with point countable bases. Examples A and B limit additions to this list.

We will discuss three other refelction properties which can be studied by the same techniques: $\mathbf{S}_{4} \equiv S\left(T_{1}\right.$, local density $\leq \omega_{1}$, countable tightness, not $\left.\mathrm{cwH}\right), \mathbf{S}_{5} \equiv S$ $\left(T_{1}\right.$, first countable, not $\left.\mathrm{cwH}\right)$, and $\mathbf{S}_{6} \equiv S$ (first countable, not metrizable). A space $X$ is said to be collectionwise Hausdorff ( $\mathrm{cwH})$ if every closed discrete subset of $X$ can be simultaneously separated by disjoint open sets. Assuming extra axioms of set theory, there are spaces similar to Examples A and B refuting the $S$ 's (§3).

In $\S 4$, Axiom $\mathrm{R}$ is explained and showns to imply $S_{1}, S_{2}$ and $S_{4}$. Further, we discuss decompositions of metric spaces. In $\S 5$, we describe iterated proper forcing collapsing a supercompact to $\omega_{2}$, and show that it makes Axiom $\mathrm{R}$ true. This forcing is flexible, and so Theorem 5.1 shows that Axiom $\mathrm{R}$ is consistent with $\mathrm{CH}$, with the Proper Forcing Axiom, with $\mathrm{MA}_{\omega_{1}}$, and with $\sim \mathrm{CH}$ and $\sim \mathrm{MA}_{\omega_{1}}$, etc. (assuming, of course, that the existence of a supercompact cardinal is consistent). Thus Axiom R cannot imply $S_{3}, S_{5}$, or $S_{6}$ because $\mathrm{MA}_{\omega_{1}}$ implies their negations.

$\S 6$ contains various remarks which do not fit elsewhere.

Set theoretic notation follows [Kun]. In particular, $|X|$ is the cardinality of $X,[X]^{<\kappa}=\{Y \subset X:|Y|<\kappa\}$, and ${ }^{A} B=\{f: f$ is a function, $\operatorname{dom} f=A$,

Received by the editors March 14, 1985.

1980 Mathematics Subject Classification. Primary 03E35, 03E55, 54D18, 54E18.

Key words and phrases. Left separated, $\sigma$-discrete, point-countable base, reflection, Martin's Axiom, proper forcing.

${ }^{1}$ Partially supported by NSF grant DMS-8401003. 
range $f \subset B\} .{ }^{<\omega} B=\bigcup\left\{{ }^{n} B: n \in \omega\right\}$. For the typist's sake, $[X]^{\omega}$ is often used for $[X]^{<\omega_{1}}$.

2. Left separated spaces with point countable bases. We call a subspace $Y$ of a space $X$ relatively discrete (respectively, weakly separated) if there is a family $\left\{U_{y}: y \in Y\right\}$ such that $U_{y}$ is a neighborhood of $y$ and if $y \neq z$ then $y \notin U_{z}$ and (respectively, or) $z \notin U_{y}$. $Y$ is closed, discrete if $Y$ is relatively discrete and closed in $X . Y$ is left separated if there is a well-ordering $<$ of $Y$ so that initial segments are closed in $Y$. A left separated space is weakly separated; consider the family $\{[y, \infty): y \in Y\}$. When $P$ is one of the above properties, $X$ is $\sigma$ - $P$ means that $X$ is a countable union of $P$ subspaces.

It is immediate that $\sigma$-closed discrete implies $\sigma$-relatively discrete implies $\sigma$ left separated implies $\sigma$-weakly separated. In a metric space, the implications can be reversed. A relatively discrete $Y$ can fail to be closed because of a set $L$ of limit points. $L$ is closed, hence $G_{\delta}$, i.e., $L=\bigcap\left\{U_{n}: n \in \omega\right\}$. Now $Y-U_{n}$ is closed, discrete, and we conclude that in perfect spaces $\sigma$-relatively discrete implies $\sigma$-closed discrete. Now let $Z$ be weakly separated by $\left\{U_{z}: z \in Z\right\}$ in a semimetric space. For each $n \in \omega$, let $Z_{n}=\left\{z \in Z: B_{n}(z) \subset U_{z}\right\}$, where $B_{n}(z)=\{y \in Z: d(y, z)<1 / n\}$. If $y, z \in Z_{n}$, then $\left(y \notin B_{n}(z)\right.$ or $\left.z \notin B_{n}(y)\right)$ because $Z$ is weakly separated and hence $\left(y \notin B_{n}(z)\right.$ and $\left.z \notin B_{n}(y)\right)$ because $d(y, z)=d(z, y)$.

We say that $X$ has point-countable base $B$ if $B$ is a base for $X$ and, for all $x \in X$, $\{B \in B: x \in B\}$ is countable. By the Nataga-Smirnov-Bing Theorem, metrizable spaces have point-countable bases. A space $X$ is said to have countable tightness if whenever $x \in X, Y \subset X$ and $x \in \bar{Y}$, then there is $a \in[Y]^{\omega}$ such that $x \in \bar{a}$. Spaces with point countable bases have countable tightness.

We define a game $G(X, B)$ of infinite length played by two players, I and II. Roughly, I aims to play a base for a point of $X$ that II does not guess. More precisely, at turn $n$, I plays $B_{n} \in B$ and II plays a countable subset, $a_{n}$, of $X$. Then they proceed to turn $n+1$. After $\omega$ turns, the winner is decided. I wins iff $\left\{B_{n}: n \in \omega\right\}$ contains a base for a point $x \in X-\bigcup\left\{a_{n}: n \in \omega\right\}$. What is a winning strategy for II? It is a function, $s$, from the set of I's previous moves to the set of plays allowed II; i.e., $s:{ }^{<\omega} B \rightarrow[X]^{\omega}$, such that if II plays according to $s$, then II wins the play of the game; i.e., if $\left(B_{n}: n \in \omega\right)$ contains a base for a point $x$, then $x \in \bigcup\left\{s\left(B_{0}, \ldots, B_{n}\right): n \in \omega\right\}$. It is possible that II has a simple winning strategy (let us call it a winning tactic, $t$ ) which looks only at I's last move and plays only one point. Rephrasing, $t: B \rightarrow X$ is such that if $\left\{B_{n}: n \in \omega\right\}$ contains a base for a point $x$, then there is $n \in \omega$, such that $t\left(B_{n}\right)=x$.

For example, if $X$ is the real line, then I can win by listing a countable base for $X$. No matter how II plays, $\bigcup\left\{a_{n}: n \in \omega\right\}$ is countable, so $X-\left\{a_{n}: n \in \omega\right\} \neq \varnothing$, and I's plays contain a base for every $x$. In the other direction, if $X$ is countable, then II can win by playing $X$, either all at one turn, or one point at a time. We leave it to the reader to work out that if $X$ is a $\sigma$-discrete metric space, then II has a winning strategy.

We say that $\mathscr{H}=\left\{H_{i}: i \in I\right\}$, a family of subsets of a space $X$ is closure preserving if for all $J \subset I, \bigcup\left\{\bar{H}_{i}: i \in J\right\}=\overline{\bigcup\left\{H_{i}: i \in J\right\}}$. If $\nVdash$ is a family of closed sets, then it is the same to say that arbitrary unions from $\mathcal{H}$ are closed. We can weaken the concept by requiring only that countable increasing unions be closed. Alone, this condition can be very weak, for maybe there are no increasing chains 
from $\nvdash$. So we add a condition yielding many increasing unions. We will express these ideas in the terminology of proper forcing. (Unfortunately, this leads us to overwork the word "closed".)

Let $\Gamma \subset[X]^{\omega}$. We say that $\Gamma$ is unbounded if for all $a \in[X]^{\omega}$ there is $c \in \Gamma$ such that $a \subset c$. We say that $\Gamma$ is closed if whenever $\left(c_{n}\right)_{n \in \omega}$ is an increasing chain from $\Gamma$, then $\bigcup\left\{c_{n}: n \in \omega\right\} \in \Gamma$. $\Gamma$ is a club if $\Gamma$ is closed and unbounded. We say that $\Sigma \subset[X]^{\omega}$ is stationary if for all clubs $\Gamma, \Sigma \cap \Gamma \neq \varnothing$. If $f:[X]^{<\omega} \rightarrow X$ and $Y \subset X$ satisfies $\left\{f(e): e \in[Y]^{<\omega}\right\} \subset Y$, we say that $Y$ is closed under $f$. The following lemma of Kueker [Kue; $\mathbf{B a}_{1}$, Theorem 1.4] is basic to proper forcing.

LEMMA 2.1. If $\Gamma \in[X]^{\omega}$ is club, then there is a function $f:[X]^{<\omega} \rightarrow X$ such that

$$
\left\{a \in[X]^{\omega}: a \text { is closed under } f\right\} \subset \Gamma .
$$

THEOREM 2.2. Let $X$ be a $T_{1}$ space with a point-countable base, B. The following are equivalent.

(a) $X$ is left separated in order type $|X|$.

(a') $X$ is $\sigma$-weakly separated.

(b) II has a winning tactic in $G(X, B)$.

(b') II has a winning strategy in $G(X, B)$.

(c) $X$ has a closure preserving cover $\nVdash=\left\{H_{i}: i \in I\right\}$ by countable closed sets.

(c') $\Gamma=\left\{a \in[X]^{\omega}: a\right.$ is closed $\}$ contains a closed unbounded subset of $[X]^{\omega}$.

Proof. The implications $(\mathrm{a}) \Rightarrow\left(\mathrm{a}^{\prime}\right),(\mathrm{b}) \Rightarrow\left(\mathrm{b}^{\prime}\right)$ and $(\mathrm{c}) \Rightarrow\left(\mathrm{c}^{\prime}\right)$ are obvious.

(a) $\Rightarrow$ (b) Let $<$ be a well-order showing that $X$ is left separated. Define $t(B)$ to be the <-least element of $B$. If $\left\{B_{n}: n \in \omega\right\}$ includes a base for $x$, then $x \in B_{n} \subset$ $[x, \infty)$ for some $n$, in which case $x=t\left(B_{n}\right)$.

$\left(\mathrm{a}^{\prime}\right) \Rightarrow\left(\mathrm{b}^{\prime}\right)$ Let $X=\bigcup\left\{X_{n}: n \in \omega\right\}$, where each $X_{n}$ is weakly separated by $\left\{U_{x}: x \in X_{n}\right\}$. For $B \in B$ define $s\left(B_{0}, \ldots, B_{m}\right)=\left\{x \in X: x \in B_{m} \subset U_{x}\right\}$. The definition of weakly separated guarantees that for each $n$, there is at most one point in $s\left(B_{0}, \ldots, B_{m}\right) \cap X_{n}$.

$\left(\mathrm{b}^{\prime}\right) \Rightarrow$ (c) For $x \in X$, we define $H_{x}$ containing $x$ such that if $B_{0}, \ldots, B_{n}$ each meet $H_{x}$, then $s\left(B_{0}, \ldots, B_{n}\right) \subset H_{x}$. Explicitly, we define countable sets $H_{x}^{k}$ and $B_{x}^{k}$ for $k \in \omega$ by induction on $k$. Set $H_{x}^{0}=\{x\}$. Set $B_{x}^{k}=\left\{B \in B: B \cap H_{x}^{k} \neq \varnothing\right\}$. $B_{x}^{k}$ is countable because $H_{x}^{k}$ is countable and $B$ is point-countable; and set $H_{x}^{k+1}=$ $\left\{s\left(B_{0}, \ldots, B_{n}\right): B_{0}, \ldots, B_{n} \in B_{x}^{k}\right\}$. Set $H_{x}=\bigcup\left\{H_{x}^{k}: k \in \omega\right\}$ and $\not t=\left\{H_{x}: x \in\right.$ $X\}$.

We have shown that $\sharp$ covers $X$ and that each $H \in H$ is countable. We will show that for all $\mathcal{G} \subset \mathcal{H} \cup \mathcal{G}$ is closed, so $\sharp$ is closure preserving. In particular, $H=$ $\bigcup\{H\}$ is closed. Let $\mathcal{G} \subset \mathcal{H}$, set $Y=\bigcup \mathcal{G}$, and let $z \in \bar{Y}$. We will show that $z \in Y$. Let $\left(B_{n}: n \in \omega\right)$ list a base for $z$. By $\left(\mathrm{b}^{\prime}\right)$ there is $n$ so that $z \in s\left(B_{0}, \ldots, B_{n}\right)$. Let $U=\bigcap\left\{B_{k}: k \leq n\right\}$; because $z \in \bar{Y}$, there is $y \in U \cap Y$. Then $y \in H_{x} \in \mathcal{G}$. If $y \in H_{x}^{k}$, then $z \in H_{x}^{k+1} \subset Y$.

$\left(c^{\prime}\right) \Rightarrow$ (a) Apply Lemma 2.1 to $\Gamma$ to obtain $f:[X]^{<\omega} \rightarrow X$. Next we claim that if $Y \subset X$ and $Y$ is closed under $f$, then $Y$ is closed. If $Y$ is countable, this follows from the choice of $f$; if $Y$ is uncountable and $z \in \bar{Y}$, by countable tightness let $a_{0} \in[Y]^{\omega}$ be such that $z \in \bar{a}_{0}$. By induction on $j \in \omega$, set $a_{j+1}=a_{j} \cup\left\{f(e): e \in\left[a_{j}\right]^{<\omega}\right\}$; $a_{j} \subset Y$ because $Y$ is closed under $f$. Now $a_{\omega}=\bigcup\left\{a_{j}: j \in \omega\right\}$ is countable and closed under $f$; hence $z \in a_{\omega} \subset Y$. 
Now we prove (a) by induction on $|Y|, Y \subset X$. If $Y$ is countable, then $Y$ is left separated because it is $T_{1}$. Now we assume that $|Y|=\lambda$ and every $Z \in[X]^{<\lambda}$ is left separated. Well order $Y=\left\{y_{\alpha}: \alpha<\lambda\right\}$. By induction, define an increasing sequence $Z_{\alpha}$ of sets closed under $f$ such that $\left\{y_{\beta}: \beta<\alpha\right\} \subset Z_{\alpha}$ and $\left|Z_{\alpha}\right|=|\alpha|$ for infinite $\alpha$. By induction hypothesis, for each $\alpha<\lambda$, there is a left separation $<_{\alpha}$ of $Z_{\alpha}$. For $y \in Y$, define $\beta(y)$ to be the least $\alpha$ such that $y \in Z_{\beta(y)}$. We define a left separation of $Y$ as follows: $y<z$ if $\beta(y)<\beta(z)$ or $\beta(y)=\beta(z)=\alpha$ and $y<_{\alpha} z$. the set of predecessors of $Y$ is $\bigcup\left\{Z_{\beta}: \beta<\alpha\right\} \cup\left\{z \in Z_{\alpha}: z<_{\alpha} y\right\}$ (where $\beta(y)=\alpha$ ), a union of two closed sets.

I thank Fred Galvin for improving this theorem. I explained to him that if $X$ has a point-countable base then the following are equivalent: $X$ is $\sigma$-left separated, $\left(\mathrm{b}^{\prime}\right)$, and a complicated statement analogous to $(c),\left(c^{\prime}\right)$. The next day Fred suggested assuming $T_{1}$, correctly formulated (c), and pointed out Corollary 2.5.

COROLlARY 2.3. If $X$ is a $T_{1}$, left separated space with a point-countable base, then for all $Y \subset X,|\bar{Y}|=Y$.

PROOF. Immediate from Theorem 2.2(c).

A more subtle corollary follows from the following theorem of Fodor [Fo; Wi, Theorem 3.1.5, p. 69].

THEOREM 2.4. Let $f: X \rightarrow[X]^{<\lambda}$. Then $X$ can be written as $\bigcup\left\{X_{\alpha}: \alpha<\lambda\right\}$ so that if $x \in X_{\alpha}$, then $f(x) \cap X_{\alpha} \subseteq\{x\}$.

COROLLARY 2.5. If $X$ is a left separated space with a point countable base, then $X$ can be written as $\bigcup\left\{X_{\alpha}: \alpha<\omega_{1}\right\}$, where each $X_{\alpha}$ is relativeiy discrete.

Proof. Let $\forall$ satisfy Theorem 2.2(c). Define $f: X \rightarrow[X]<\omega_{1}$ so that $x \in$ $f(x) \in \mathcal{H}$. Apply Fodor's Theorem. If $x \in X_{\alpha}$, then $K=\bigcup\left\{f(y): y \in X_{\alpha}-\{x\}\right\}$ is closed, and $(X-K) \cap X_{\alpha}=\{x\}$.

We conclude this section with some results about concepts related to those mentioned in Theorem 2.2.

LEMMA 2.6. If $X$ is a left separated $T_{1}$ space with a $\sigma$-point-finite base $B=$ $\bigcup\left\{B_{n}: n \in \omega\right\}$, then $X$ is $\sigma$-relatively discrete.

Proof. Let $<$ left separate $X$. For $x \in X$, choose $U_{x} \in B$, so that $x \in U_{x} \subset$ $[x, \infty)$. Set $X_{n}=\left\{x \in X: U_{x} \in B_{n}\right\}$. Fix $n \in \omega$. Set $Y_{0}=\varnothing$ and

$$
Y_{j+1}=\left\{y \in X_{n}: \text { if } x<y \& x \in X_{n}-\bigcup\left\{Y_{k}: k \leq j\right\}, \text { then } y \notin U_{x}\right\} \text {. }
$$

Because $B_{n}$ is point-finite, $X_{n}=\bigcup\left\{Y_{j}: j<\omega\right\}$.

LEMMA 2.7. (a) If $X$ has a closure preserving cover by finite sets, then $X$ is $\sigma$-relatively discrete.

(b) If $X$ is $\sigma$-relatively discrete, perfect, and collectionwise Hausdorff, then $X$ has a closure preserving cover by finite sets.

Proof. (a) As in Corollary 2.5. Theorem 2.4 with $\lambda=\omega$ is due to Erdös and DeBrujn.

(b) By perfect and $\sigma$-relatively discrete, $X=\bigcup\left\{X_{n}: n \in \omega\right\}$ where each $X_{n}$ is closed, discrete. Apply collectionwise Hausdorff to obtain disjoint open families $\mathcal{U}_{n}=\left\{U_{x}: x \in X_{n}\right\}$ with $x \in U_{x}$ and $U_{x} \cap\left(\bigcup\left\{X_{k}: k \leq n\right\}\right)=\{x\}$. We define an 
open set $V_{k}$ containing $x$ for $x \in X_{n}$ by induction on $n$. For $x \in X_{0}$, set $V_{x}=U_{x}$. For $x \in X_{n}, n>0$, set

$$
\begin{aligned}
& F_{x}=\left\{y \in X_{k}: k<n \text { and } x \in V_{y}\right\}, \\
& V_{x}=U_{x} \cap\left(\bigcap\left\{V_{y}: y \in F_{x}\right\}\right), \\
& H_{x}=F_{x} \cup\{x\} .
\end{aligned}
$$

It is routine to check that $y \in H_{x}$ iff $x \in V_{y}$. We claim that $\left\{H_{x}: x \in X\right\}$ is closure preserving. Suppose that $z \in \bar{U}\left\{H_{x}: x \in X^{\prime}\right\}$; then $y \in H_{x} \cap V_{z}$ for some $x \in X^{\prime}, y \in X$. Hence $x \in V_{x} \subset V_{y} \subset V_{z}$ and $z \in H_{x}$.

The examples in the following section show that Lemma 2.7 cannot be improved to an equivalence. We may delete from the hypothesis of 2.7(b) neither perfect (Michael line), nor cwH (Cantor tree). The existence of a closure preserving cover by finite sets implies neither perfect (one point compactification of uncountable discrete space) nor cwH (Pixley-Roy).

\section{Examples.}

EXAMPLE A. Let $\Lambda$ be the set of countable limit ordinals. For $\delta \in \Lambda$, let $\eta_{\delta}: \omega \rightarrow \delta$ be increasing and cofinal in $\delta$. Define a metric $d$ on $\Lambda$ by $d(\delta, \zeta)=2^{-n}$, where $n$ is least so that $\eta_{\delta}\left|n \neq \eta_{\zeta}\right| n$. The conclusion of Corollary 2.3 holds because if $Y \subset \Lambda$, then $\sup \bar{Y}=\sup Y$. The conclusion of Corollary 2.5 holds because each $\{\delta\}$ is relatively discrete. A point-countable base is $B=\left\{B_{\sigma}: \sigma \in^{<\omega} \omega_{1}\right\}$, where $B_{\sigma}=\left\{\delta \in \Lambda: \eta_{\delta} \mid n=\sigma\right\}$. If $\delta \in B_{\sigma}$, then max range $\sigma<\delta$, so the pressing down lemma yields that $\Lambda$ is not left separated. In fact, Theorem 2.2(b) is strongly contradicted.

\section{LEMMA 3.1. Player I has a winning strategy in $G(\Lambda, B)$.}

ProOF. It is easy to verify that the following changes in $G(\Lambda, B)$ do not change the existence of a winning strategy: (1) player I may play countably many basic open sets at each turn; (2) at turn $n+1$ player II must play $a_{n+1} \supset a_{n}$ such that $\sup a_{n+1}>\sup a_{n}$. (If $a_{n}$ is enlarged I's task is harder. I can in effect play countably many moves at once using a partition of $\omega$ into infinitely many infinite pieces.) Now a strategy for $I$ is to play $\left\{B_{\sigma}: \operatorname{range} \sigma \subset \sup a_{n}\right\}$ at $\operatorname{turn} n+1$. After $\omega$ steps, I's plays include a base for $\sup \left\{a_{n}: n \in \omega\right\} \notin \bigcup\left\{a_{n}: n \in \omega\right\}$.

For subspaces $Y$ of $\Lambda$, I has a winning strategy in $G(Y, B)$ iff $Y$ contains a club, II has a winning strategy in $G(Y, B)$ iff $Y$ is nonstationary. Hence the game is undetermined if both $Y$ and $\omega_{1}-Y$ are stationary.

The following axiom is true in $L$, every generic extension of $L$, and every model of set theory without inner models of many large cardinals (see [KM, p. 222]).

AxIOM E. For some regular cardinal $\lambda>\omega_{1}$, there is $E \subset\{\delta \in \lambda: \operatorname{cf} \lambda=\omega\}$ stationary in $\lambda$ with $E \cap \beta$ nonstationary in $\beta$ for all $\beta<\lambda$.

ExAmple A1. Assume $\lambda, E$ satisfy Axiom E. For $\delta \in E$, let $\eta_{\delta}: \omega \rightarrow \delta$ be increasing and cofinal in $\delta$. Define a metric $d$ on $E$ by $d(\delta, \zeta)=2^{-n}$, where $n$ is least so that $\eta_{\delta}\left|n \neq \eta_{\zeta}\right| n$. Then $E$ is not $\sigma$-discrete, but every $Y \in[E]^{<\lambda}$ is $\sigma$-discrete (see [Po]). Thus Axiom E implies that $S_{1}, S_{2}$ and $S_{3}$ fail.

EXAMPLe A2. Assume $\lambda, E$ satisfy Axiom E; define $\eta_{\delta}$ as above. Let $Z=$ $E \cup{ }^{<\omega} \lambda$. Let ${ }^{<\omega} \lambda$ be the set of isolated points. Define the $m$ th basic open set of $\delta \in E$ to be $\{\delta\} \cup\left\{\eta_{\delta} \mid n: m \leq n\right\}$. Then $Z$ is first countable and locally countable 
but not cwH. Every $Y \in[Z]^{<\lambda}$ is $\mathrm{cwH}$ and metrizable (see $\left[\mathbf{F}_{\mathbf{3}}\right]$ ). Thus Axiom $\mathrm{E}$ implies that $S_{4}, S_{5}$ and $S_{6}$ fail.

EXAMPLE B. This space is from Aull [Au], who calls it a modification of an example of Miščenko [Mi]. I thank Pete Nyikos for calling this example to my attention.

Let $F=\bigcup\left\{{ }^{\alpha} \omega: \alpha \in \omega_{1}\right\}$. Basic open sets are indexed by $f \in F, n \in \omega$ :

$$
B(f, n)=\{f\} \cup\{g \in F: f \subset g \text { and } g(\operatorname{dom} f) \geq n\} .
$$

We can picture $F$ as a tree where open sets "look up".

$$
\begin{aligned}
& \text { If } B(f, n) \cap B(g, m) \neq \varnothing, \\
& \text { then either } f \subset g \text { and } B(f, n) \supset B(g, m) \\
& \text { or } g \subset f \text { and } B(g, m) \subset B(f, n) .
\end{aligned}
$$

It is routine to verify that $F$ is a $T_{1}$, regular, ultraparacompact, left separated space. (Given an open cover $\mathcal{U}$, define a disjoint refinement by induction on $\operatorname{dom} f-$ possible by $(*)$.)

\section{LEMMA 3.2. $F$ is not $\sigma$-relatively discrete.}

PROOF. Towards a contradiction, assume that $\left\{F_{n}: n \in \omega\right\}$ and $\theta: F \rightarrow \omega$ are such that $F=\bigcup\left\{F_{n}: n \in \omega\right\}$ and, for each $n \in \omega,\left\{B(f, \theta(f)): f \in F_{n}\right\}$ is disjoint. By induction on $\alpha<\omega_{1}$, define $f_{\alpha} \in{ }^{\alpha} \omega$ so that if $\alpha<\beta$, then $f_{\beta}(\alpha)=\theta\left(f_{\alpha}\right)$, hence $f_{\beta} \in B\left(f_{\alpha}, \theta\left(f_{\alpha}\right)\right)$. For some $n \in \omega$ and $\alpha<\beta<\omega_{1}, f_{\alpha}, f_{\beta} \in F_{n}$ but $f_{\beta} \in B\left(f_{\alpha}, \theta\left(f_{\alpha}\right)\right)$. Contradiction.

Hence $\sigma$-relatively discrete cannot be added to the list in Theorem 2.2.

Next, we assume that the weak Kurepa Hypothesis fails $(\sim \mathrm{wKH})$ and Martin's Axiom ( $\left.\mathrm{MA}_{\omega_{1}}\right)$ holds (see [ $\mathbf{T o}_{\mathbf{1}}$ and $\mathbf{B a}$, Theorem 7.10]).

LEMMA 3.3. $\left(\mathrm{MA}_{\omega_{1}}+\sim \mathrm{wKH}\right)$. Every $Y \in[F]^{\omega_{1}}$ is $\sigma$-closed discrete, and hence metrizable.

PROOF. If $Y \in[F]^{\omega_{1}}$, then $Y$ is a tree of height and cardinality $\omega_{1}$, so $Y$ has at most $\omega_{1}$ uncountable branches, $\left\{b_{\alpha}: \alpha<\omega_{1}\right\}$ (this is $\sim \mathrm{wKH}$ ). By induction on $\alpha<\omega_{1}$ we can define $c_{\alpha}$ as a final segment of $b_{\alpha}$ so that $\left\{c_{\alpha}: \alpha \in \omega_{1}\right\}$ is disjoint. We consider $T=Y-\bigcup\left\{c_{\alpha}-\left\{\min c_{\alpha}\right\}: \alpha<\omega_{1}\right\}$. T has no $\omega_{1}$-branches, so by $\mathrm{MA}_{\omega_{1}}$ [BMR], $T$ is the union of countably many antichains; i.e., $T=\bigcup\left\{A_{n}: n \in \omega\right\}$. Let $Y_{n}=A_{n} \cup \bigcup\left\{c_{\alpha}: \min c_{\alpha} \in A_{n}\right\}$. Then $Y=\bigcup\left\{Y_{n}: n \in \omega\right\}$ and if $y \in Y_{n}$ then $\{f \in Y: y \subset f\}$ is totally ordered. Hence each $Y_{n}$ is relatively discrete.

Fix $\theta: Y \rightarrow \omega$ so that $\left\{B(y, \theta(y)): y \in Y_{n}\right\}$ is disjoint for all $n \in \omega$. For each $n \in \omega$ we define $P_{n}$, a poset of finite approximations to a closed subset of $Y_{n}$. Let $P_{n}$ be the set of triples $(a, s, \eta)$ satisfying

(1) $a \in\left[Y_{n}\right]<\omega$,

(2) $s \in\left[Y-Y_{n}\right]<\omega$,

(3) $\eta: s \rightarrow \omega$,

(4) $\forall y \in s, \eta(y) \geq \theta(y)$,

(5) $\forall y \in s, B(y, \eta(y)) \cap a=\varnothing$.

We show that $P_{n}$ is ccc. Aiming for a contradiction, suppose that $W \in\left[P_{n}\right]^{\omega_{1}}$ is a set of pairwise incompatible elements. By usual counting arguments $(\Delta$-system lemma, discard the root, etc. $)$, we may assume that $W=\left\{\left(a_{\alpha}, s_{\alpha}, \eta_{\alpha}\right): \alpha \in \omega_{1}\right\}$ satisfies for some fixed $k \in \omega, l \in \omega$ and $m: l \rightarrow \omega$, 
(1) $\left\{a_{\alpha} \cup s_{\alpha}: \alpha \in \omega_{1}\right\}$ is disjoint,

(2) $\forall \alpha \in \omega_{1}, a_{\alpha}=\{a(\alpha, i): i<k\}$,

(3) $\forall \alpha \in \omega_{1}, s_{\alpha}=\{s(\alpha, j): j<l\}$,

(4) $\forall \alpha \in \omega_{1} \forall_{j}<l, s(\alpha, j) \in Y_{m(j)}$.

Because $W$ is pairwise incompatible, we can define $\Phi:\left[\omega_{1}\right]^{2} \rightarrow k \times l \times\{0,1\}$ so that if $\Phi(\alpha, \beta)=(i, j, e)$, then $a(\beta, i) \in B(s(\alpha, j), \eta(s(\alpha, j)))$ and $\alpha<\beta$ if $e=0$, $\beta<\alpha$ if $e=1$. Apply Ramsey's Theorem to get $i<k, j<l, e<2$ and $\alpha, \beta, \gamma<\omega$ so that

$$
a(\gamma, i) \in B(s(\alpha, j), \eta(s(\alpha, j))) \cap B(s(\beta, j), \eta(s(\beta, j))),
$$

and $\alpha<\beta<\gamma$ if $e=0, \gamma<\beta<\alpha$ if $e=1$. This contradicts that $\{B(y, \theta(y)): y \in$ $\left.Y_{m(j)}\right\}$ is disjoint.

By $\mathrm{MA}_{\omega_{1}}, P=\left(P_{n}\right)^{\omega}$, the finite support product of $\omega$ copies of $P_{n}$, has ccc. An element of $P$ can be considered to be a triple of functions, $(a, s, \eta)$ with domain $\omega$ so that $(a(i), s(i), \eta(i)) \in P_{n}$ and $\{i:(a(i), s(i), \eta(i)) \neq(\varnothing, \varnothing, \varnothing)\}$ is finite for all $i$. For each $y \in Y_{n}, D_{y}=\{(a, s, \eta):(\exists i)(y \in a(i))\}$ is dense. For each $i \in \omega$ and $f \in Y-Y_{n}, D_{i f}=\{(a, s, \eta): f \in s(i)\}$ is dense. By MA $_{\omega_{1}}$, let $G$ be a filter on $P$ meeting each of the above dense sets. Set $Z_{i}=\bigcup\{a(i):(a, s, \eta) \in G\}$. Then each $Z_{i}$ is closed, discrete and $Y_{n}=\bigcup\left\{Z_{i}: i \in \omega\right\}$. Because this construction can be done for all $n \in \omega, Y$ is $\sigma$-closed discrete. Then by $(*)$ and Nagata-Smirnov-Bing, $Y$ is metrizable.

Thus, $\mathrm{MA}_{\omega_{1}}+\sim$ wKH implies that $S_{3}$ and $S_{6}$ fail.

Choose $A \subset \omega_{1}$ with both $A$ and $\omega_{1}-A$ stationary. Let $U(A)$ be the tree of continuous, increasing functions from a countable ordinal to $A$. Gary Gruenhage suggested using $U(A)$ in place of $F$ in Example B. Because $U(A)$ has no $\omega_{1}$-branches, we do not need $\sim$ wKH before applying $\mathbf{M A}_{\omega_{1}}$ and [BMR]. This space is essentially the same as the linearly ordered space $M(A)$ of maximal branches through $U(A)$ (see $\left[\mathbf{T o}_{2}\right.$, p. 288]). An analog of Example B1 also can be constructed. Thus $\mathrm{MA}_{\omega_{1}}$ suffices to refute $S_{3}, S_{5}$ and $S_{6}$.

EXAMPle B1. Assume $\sim \mathrm{wKH}+\mathrm{MA}_{\omega_{1}}$. Let $F$ be as in Example B. For each $f \in F$, enumerate $\{h \in F: h \subset f\}$ as $\{h(f, m): m<\omega\}$ and set $e(f, n)=$ $\{h(f, m): m \leq n\}$. Let $W=\left\{(f, g) \in F^{2}: f \subset g\right\}$. Set $X=F \cup W$. Points of $W$ are isolated. The other basic open sets are indexed by $f \in F$ and $n \in \omega$ :

$$
U(f, n)=\{f\} \cup\{(f, g) \in W: g(\operatorname{dom} f) \geq n\} \cup\{(h, f) \in W: h \notin e(f, n)\} .
$$

With this basis $X$ is a Moore space and $F$ is closed discrete. If $A \in[F]^{\omega_{1}}$, then $A$ is $\sigma$-closed discrete in the topology of Example B. It is routine to use that fact to separate $A$ in $X$.

$X$ is not cwH because $F$ cannot be separated. We follow the proof of Lemma 3.2. Towards a contradiction, assume that $\theta: F \rightarrow \omega$ is such that $\{U(f, \theta(f)): f \in F\}$ is disjoint. By induction on $\alpha<\omega_{1}$, define $f_{\alpha} \in{ }^{\alpha} \omega$ so that if $\alpha<\beta$, then $f_{\beta}(\alpha) \geq \theta\left(f_{\alpha}\right)$ : i.e., $\left(f_{\alpha}, f_{\beta}\right) \in U\left(f_{\alpha}, \theta\left(f_{\alpha}\right)\right)$. By the pressing down lemma, there is $n \in \omega, e \in\left[\omega_{1}\right]^{<\omega}$ and a stationary set $S$ such that if $\alpha \in S$, then $\theta\left(f_{\alpha}\right)=$ $n$ and $e\left(f_{\alpha}, n\right)=e$. If $\alpha, \beta \in S$, then $\left(f_{\alpha}, f_{\beta}\right) \in U\left(f_{\alpha}, \theta\left(f_{\alpha}\right)\right) \cap U\left(f_{\beta}, \theta\left(f_{\beta}\right)\right)$. Contradiction.

EXAMPLE C. This example is from [ $\mathbf{v D}$, Remark 12.6], to which the reader is referred for more details and more information about $\mathfrak{b}$. The point set of $C$ is $\left({ }^{\omega} \omega\right) \cup \omega \cup\left({ }^{\omega} \omega \times \omega \times \omega\right)$. Points of ${ }^{\omega} \omega \times \omega \times \omega$ are isolated. The $n$th neighborhood 
of $k \in \omega \subset C$ is $\{k\} \cup\left({ }^{\omega} \omega \times\{k\} \times(\omega-n)\right)$. The $n$th neighborhood of $f \in{ }^{\omega} \omega \subset C$ is $\{f\} \cup(\{f\} \times\{(k, f(k)): k \geq n\})$.

Consider subspaces $C(H)$ of the form $H \cup \omega \cup(H \times \omega \times \omega)$, where $H \subset \omega_{\omega}$. If $H$ is bounded, i.e., there is $f \in \omega^{\omega} \omega$ such that for all $h \in H,\{n \in \omega: f(n) \leq h(n)\}$ is finite, then $C(H)$ is metrizable. If $H$ is unbounded, then $C(H)$ is not cwH. Hence $\mathfrak{b}>\omega_{1}$ (i.e., every $H \in\left[{ }^{\omega} \omega\right]^{\omega_{1}}$ is bounded) implies that $S_{5}$ and $S_{6}$ fail.

4. Axiom $\mathbf{R}$ and applications. We have seen in $\S 3$ that Axiom $E$ implies that all the $S_{i}$ 's fail. Since Axiom $E$ implies the existence of counterexamples, $\sim E$, the negation of Axiom $\mathrm{E}$ is potentially weaker than the $S_{i}$ 's. In any case, it is often frustrating to work with $\sim E$ because it is so "linear". (An exception: it follows quickly from Engelking and Lutzer [EL] that $S$ (first countable linearly ordered topological space, not paracompact) is equivalent to $E$.)

The statement that stationary subsets of $[X]^{\omega}$ reflect is stronger than the statement that stationary subsets of $\{\alpha \in \kappa: \operatorname{cf} \alpha=\omega\}$ reflect. Even this seems to be not enough because if $X$ has structure, we may need to reflect to a closed subset of $X$.

Recall that a space $X$ has countable tightness when for all $x \in X$ and $\bar{Y} \in X$, if $x \in \bar{Y}$, then $\exists a \in[Y]^{\omega} x \in \bar{a}$. It is easy to see that in a space of countable tightness, the union of an increasing sequence of closed sets is closed if the sequence has uncountable cofinality. We say that $\Gamma \subset[X]^{<\kappa}$ is tight if whenever $\left\{C_{\alpha}: \alpha<\delta\right\}$ is an increasing sequence from $\Gamma$ and $\omega<\operatorname{cf} \delta<\kappa$, then $\bigcup\left\{C_{\alpha}: \alpha<\delta\right\} \in \Gamma$.

AxIOM R. If $\Sigma \subset[X]^{\omega}$ is stationary and $\Gamma \subset[X]^{<\omega_{2}}$ is tight and unbounded, then there is $Y \in \Gamma$ such that $P(Y) \cap \Sigma$ is stationary in $[Y]^{\omega}$.

LEMMA 4.1. Let $X$ be a $T_{1}$ space with a point countable base. If every $Y \in$ $[X]^{<\omega_{2}}$ is left separated, then for all $Y \in[X]^{<\omega_{2}},|\bar{Y}|=|Y|$. Hence $\Gamma=\{Y \in$ $[X]^{<\omega_{2}}: Y$ is closed $\}$ is tight and unbounded.

Proof. For $Y$ finite, use $T_{1}$. Let $|Y|=\omega$. Towards a contradiction, assume that $|\bar{Y}|>\omega$, choose $Z \in[X]^{\omega_{1}}$ with $Y \subset Z \subset \bar{Y}$. Then $\mid$ closure $_{Z}(Y)|=| \bar{Y} \cap Z \mid=$ $|Z|=\omega_{1}$. However $Z$ is left separated, so by Corollary $2.3\left|\operatorname{closure}_{Z}(Y)\right|=\omega$. Now let $Y=\left\{y_{\alpha}: \alpha<\omega_{1}\right\}$. By countable tightness, $\bar{Y}=\bigcup\left\{\left\{\overline{y_{\beta}: \beta<\alpha}\right\}: \alpha<\omega_{1}\right\}$. Hence $|\bar{Y}|=\omega_{1} \cdot \omega=\omega_{1}$.

$\Gamma$ is tight because $X$ has countable tightness. $\Gamma$ is unbounded because for all $Y \in[X]^{<\omega_{2}}, Y \subset \bar{Y} \in \Gamma$.

\section{THEOREM 4.2. Axiom R implies $S_{2}$ (hence $S_{1}$ ).}

PROOF. Towards a contradiction, assume (a) $X$ is a $T_{1}$ not left separated space with a point countable base and (b) every $Y \in[X]^{<\omega_{2}}$ is left separated. From (a) and Theorem 2.2( $\left.\mathrm{c}^{\prime}\right)$ we get that $\Sigma=\left\{a \in[X]^{\omega}: a\right.$ is not closed in $\left.X\right\}$ is stationary in $[X]^{\omega}$. From (b) and Lemma 4.1 we get that $\Gamma=\left\{Y \in[X]^{<\omega_{2}}: Y\right.$ is closed $\}$ is tight and unbounded. Applying Axiom $\mathrm{R}$, we get $Y \in \Gamma$ such that $\Sigma \cap[Y]^{\omega}=\left\{a \in[Y]^{\omega} ; a\right.$ is not closed in $\left.X\right\}=\left\{a \in[Y]^{\omega}: a\right.$ is not closed in $\left.Y\right\}$ is stationary in $[Y]^{\omega}$. (The second equality is why we wanted $Y$ to be closed.) Hence $Y \in[X]^{<\omega_{2}}$ is not left separated. Contradiction.

In order to use the technique of the above proof for other properties, we must first have a characterization of those properties in terms of $[X]^{\omega}$. We give three 
more examples. (Forcing and reflection proofs of the consistency of 4.4 and 4.7 are well known.)

Let $T$ be a tree of height $\omega$ and $B$ a set of infinite branches through $T$. We say that $B$ is nonstationary if there exists a one-to-one function $f: B \rightarrow T$ such that for all $b \in B, f(b) \in b$. $B$ is stationary otherwise. (This terminology is justified by Theorem 4.3.) Set $X=T \cup B$. Say that $Y \subset X$ is $B$-closed iff (i) for all $b \in B$, $b \subset Y$ implies $b \in Y$ and (ii) $s<_{T} t$ and $t \in Y$ implies $s \in Y$. The set of $B$-closed sets is tight.

THEOREM 4.3. Let $T$ be a tree of height $\omega$ and $B$ a set of infinite branches through $T . B$ is stationary if and only if $\left\{a \in[T \cup B]^{\omega}: a\right.$ is closed $\}$ is stationary.

Proof. This is Theorem 8.5 of $\left[\mathbf{B a}_{1}\right]$, where the proof is done in detail. The only if direction is similar to $\left(\mathrm{c}^{\prime}\right) \Rightarrow$ (a) of Theorem 1 , by induction on cardinality of $X=T \cup B$ and using a function $f:[X]^{<\omega} \rightarrow X$.

COROLlARY 4.4 (AXIOM R). If $B$ is a stationary set of branches through a tree $T$ of height $\omega$, then there is a stationary $A \in[B]^{<\omega_{2}}$.

ProOF. First prove the analogue of 4.1, then follow the proof of 4.2.

We can show, again by induction on $|X|$, that $B$ is nonstationary iff there is a function $f^{\prime}: B \rightarrow T$ so that $f^{\prime}(b) \in b$ and $\{\{t \in b: f(b) \leq t\}: b \in B\}$ is disjoint. If we topologize $X$ so that each $t \in T$ is isolated, and a neighborhood of $b \in B$ has the form $\{b\} \cup\{t \in b: s \leq t\}$ for some $s \in b$, then $X$ is a space similar to Examples B1 and B2. Moreover $B$ is nonstationary iff $X$ is cwH. Generalizing from trees of height $\omega$, Shelah $\left[\mathbf{S h}_{2}\right]$ showed $S$ (locally countable, not $\mathrm{cwH}$ ) holds after the Levy collapse of a supercompact to $\omega_{2}$. However, locally countable spaces are very special. Manifolds, which are more geometric, are locally separable and have countable tightness. These ideas lead to the unusual hypotheses of Lemma 4.5. (Also see recent work of Tall, e.g. $\left[\mathbf{T}_{\mathbf{2}}\right]$.)

LEMMA 4.5. Let $D$ be a closed discrete subset of a space $X$ and $\kappa$ a cardinal such that

(a) $\forall A \in[D]^{<\kappa}, A$ can be separated.

(b) $\exists \beta<\kappa \forall y \in D$, $y$ has a neighborhood of density $\leq \beta$,

(c) $X$ has countable tightness at every point of $D$ (i.e.,

$$
\left.(\forall y \in D \forall Z \subset X)\left(y \in \bar{Z} \rightarrow \exists a \in[Z]^{\omega}\right)(y \in \bar{a})\right) .
$$

Then $D$ can be separated iff $\left\{a \in[X]^{\omega}: \bar{a} \cap D \neq a \cap D\right\}$ is not stationary.

Proof. $(\rightarrow)$ is easy.

$(\leftarrow)$ Say that $Z \subset X$ is $D$-closed if $\bar{Z} \cap D=Z \cap D$. By Lemma 1.1, let $f:[X]^{<\omega} \rightarrow X$ be such that closed under $f$ impiies $D$-closed. By (b), for $y \in D$, choose $Q_{y} \in[X] \leq \beta$ so that $y \in \operatorname{Int}\left(\bar{Q}_{y}\right)$. The proof is by induction on $X$, again. By (a) $D$ can be separated if $|X|<\kappa$. For $|X| \geq \kappa$, we can define a continuous increasing sequence of sets, $X_{\alpha}$, so that $\bigcup\left\{X_{\alpha}: \alpha<|X|\right\}=X,\left|X_{\alpha}\right|<|X|$, and each $X_{\alpha}$ is $D$-closed and $Q$-closed; (i.e., $y \in X_{\alpha}$ implies $Q_{y} \subset X_{\alpha}$ ). By inductifon hypothesis, for each $\alpha, D_{\alpha}=D \cap\left(X_{\alpha+1}-X_{\alpha}\right)$ can be separated in $X_{\alpha+1}$-by $\left\{U_{y}: y \in D_{\alpha}\right\}$, say. Then

$$
\bigcup\left\{\left\{\operatorname{Int}\left(\bar{U}_{y}\right)-\bar{X}_{\alpha}: y \in D_{\alpha}\right\}: \alpha<|X|\right\}
$$

separates $D$ in $X$. 
COROllary 4.6 (AXIOM R). If $X$ is $\omega_{1}-\mathrm{cwH}$, has local density $\omega_{1}$, and has countable tightness, then $X$ is $\mathrm{cwH}$. In particular $\omega_{1}-\mathrm{cwH}$ manifolds are $\mathrm{cwH}$.

Proof. Again, prove the analogue of 4.1 and follow the proof of 4.2.

A graph $G=(V, E)$ consists of a set $V$ of vertices and a set of edges $E \subset[V]^{2}$. $G$ is said to have coloring number $\leq \omega$ if there is a well-ordering $<$ of $V$ so that for all $v \in V,\{u \in V:\{u, v\} \in E\}$ is finite.

THEOREM 4.7 (AXIOM R). If $G=(V, E)$ is a graph such that $\left(Y, E \cap[Y]^{2}\right)$ has coloring number $\leq \omega$ for every $Y \in[V]^{<\omega_{2}}$, then $G$ has coloring number $\leq \omega$.

Proof. Say that $Y \subset V$ is $E$-closed if for all $v \in V,\{y \in Y:(y, v) \in E\}$ infinite implies $v \in Y$. Argue as above.

Below, we will use Axiom $\mathrm{R}$ to prove a reflection property which fits well with theorems of $\mathrm{Pol}[\mathbf{P o}]$ and Hansell $[\mathbf{H a}]$ on $\sigma$-discretely refinable families. Analogous results about $\sigma$-discretely decomposible families are left to the reader. These results allow us to replace Axiom SC $\left(\omega_{2}\right)$ with Axiom R in Lemma 4.9 of $\left[\mathbf{F}_{1}\right]$. For further discussion and references, see $[\mathbf{F r}]$.

Let $\mathcal{E}=\left(E_{\xi}: \xi \in I\right)$ be an indexed family of subsets of a metric space $X$ with $\sigma$-discrete base $B=\bigcup\left\{B_{n}: n \in \omega\right\}$. We will assume that $X, I$ and $B$ are pairwise disjoint. We say that $\mathcal{E}$ is $\sigma$-discretely refinable if there exists an indexed family $\mathcal{E}^{\prime}=\left(E_{\xi j}: \xi \in I, j \in \omega\right)$ such that (a) $\forall j \in \omega,\left(E_{\xi j}: \xi \in I\right)$ is discrete, (b) $\forall_{\xi} \in I \forall j \in \omega, E_{\xi j} \subset E_{\xi}$, and (c) $\bigcup \mathcal{E}^{\prime}=\bigcup \mathcal{E}$. We say that $a \subset B \cup I$ is $\mathcal{E}$-closed if whenever $a \cap B$ contains a base for $x \in \bigcup \mathcal{E}$, then $x \in \bigcup\left\{E_{\xi}: \xi \in a \cap I\right\}$.

LEMMA 4.8. Let $\mathcal{E}, X, I$ and $B$ be as above. The following are equivalent.

(a) $\mathcal{E}$ is $\sigma$-discretely refinable.

(b) There is $s: B \rightarrow[I]^{\omega}$ such that for all $b \in[B]^{\omega}, b \cup(\bigcup\{s(B): B \in b\}$ is $\mathcal{E}$-closed.

(c) $\left\{a \in[B \cup I]^{\omega}: a\right.$ is $\mathcal{E}$-closed $\}$ contains a club subset $\Gamma \subset[B \cup I]^{\omega}$.

ProOF. The proof of this analogue to 2.2 is routine except for $(c) \Rightarrow(b)$. Given $\Gamma$, let $f:[B \cup I]^{<\omega} \rightarrow B \cup I$ be the Kueker function. For $u \in[B]^{<\omega}$ and $j \in \omega$, define $u_{0}=u, u_{j+1}=u_{j} \cup$ range $f \mid\left[u_{j}\right]^{<\omega}$ and $u^{f}=\bigcup\left\{u_{j}: j \in \omega\right\}$. (Compare $2.2\left(\mathrm{c}^{\prime}\right) \Rightarrow(\mathrm{a})$.)

For $B \in B$, let

$$
U(B)=\left\{\left\{B_{0}, B_{1}, \ldots, B_{m}\right\} \in[B]^{<\omega}: B_{1} \supset B_{1} \supset \cdots \supset B_{m}=B\right\}
$$

be a countable set. Set $s(B)=\bigcup\left\{u^{f}: u \in U(B)\right\}$.

Towards verifying (b), let $\left\{B_{k}: k \in \omega\right\}$ contain a base for $x \in \bigcup \mathcal{E}$. By passing to a subsequence we may assume that $B_{0} \supset B_{1} \supset \cdots \supset B_{m} \supset \cdots$. (The modifications in case $x$ is isolated are easy.) For each $m \in \omega$, set $a_{m}=\left\{B_{0}, \ldots, B_{m}\right\}^{f}$; then $\left\{B_{k}: k \in \omega\right\} \subset \bigcup\left\{a_{m}: m \in \omega\right\} \in \Gamma$. Hence for some $m \in \omega, x \in a_{m} \subset s\left(B_{m}\right)$.

For $Y \subset X$, set $\mathcal{E} \mid Y=\left(E_{\xi} \cap Y: \xi \in I\right)$ and $I(Y)=\left\{\xi \in I: E_{\xi} \cap Y \neq \varnothing\right\}$. We say that $\mathcal{E}$ is $\omega_{1}$-like if whenever $Y \subset X$ has weight $\leq \omega_{1},|I(Y)| \leq \omega_{1}$. If $\mathrm{CH}$ holds, then point countable families are $\omega_{1}$-like. If $C \subset B$, set $Y(C)=\{x \in \bigcup \mathcal{E}$ : $C$ contains a base for $x$ \}.

COROLlaRY 4.9 (AXIOM R). Let $X, \mathcal{E}$ be as above. If $\mathcal{E}$ is $\omega_{1}$-like and not $\sigma$-discretely refinable, then there is $Y \subset X$, weight $Y \leq \omega_{1}$ such that $\mathcal{E} \mid Y$ is not $\sigma$-discretely refinable. 
Proof. Set $\Gamma=\left\{a \in[B \cup I]^{<\omega_{2}}: A\right.$ is $\mathcal{E}$-closed and $\left.I(Y(A \cap B)) \subset I \cap A\right\}$. Argue as in 4.2.

Axiom $\forall S \diamond_{s}$ is a consequence of $V=L$, implies $\mathrm{CH}$ and is consistent with Axiom R. The proof of the next result follows ideas in $\left[\mathbf{F}_{\mathbf{1}}, \mathbf{H a}\right.$, and $\left.\mathbf{F r}\right]$.

Corollary 4.10 (AXIOM $\mathrm{R}+\forall S \diamond_{s}$ ). Let $X, \mathcal{E}=\left(E_{\xi}: \xi \in I\right)$ be as above. If $\mathcal{E}$ is point-countable and for all $J \subset I, \bigcup\left\{E_{\xi}: \xi \in J\right\}$ is Borel in $X$, then $\mathcal{E}$ is $\sigma$-discretely refinable.

5. Properly collapsing a supercompact cardinal. We will force with complete Boolean algebras, B. A B-name is a function from (already defined) B-names to $\mathbf{B}$. For definition and dicussion of proper forcing, see $\left[\mathbf{B} \mathbf{a}_{1}\right]$. An iterated proper forcing is a sequence $\left(\mathbf{B}_{\alpha}: \alpha \leq \kappa\right)$ such that for $\alpha<\beta, \mathbf{B}_{\alpha}$ is a complete subalgebra of $\mathbf{B}_{\beta}$ so that a $\mathbf{B}_{\alpha}$ name is a $\mathbf{B}_{\beta}$ name (see, e.g. $\left[\mathbf{B a}_{2}\right]$ ). Moreover if $\nu \leq \kappa$ is inaccessible, then $\mathbf{B}_{\nu}=\bigcup\left\{\mathbf{B}_{\alpha}: \alpha<\nu\right\}$ is proper and $\mathbf{B}_{\kappa}=\mathbf{B}_{\nu} * \dot{\mathbf{A}}_{\nu}$, where $\mathbf{B}_{\nu}$ is proper and $\mathbf{A}_{\nu} \in V^{\mathbf{B}_{\nu}}$ is proper in $V^{\mathbf{B}_{\nu}}$.

Let $\mathbf{B}$ have $\kappa c c$, where $\kappa$ is regular. If $X \in V$ and $A \in[X]^{<\kappa} \cap V[G]$ (where $G$ is $V$-generic on B), then $A$ can be represented in $V$ by a name in $\mathcal{N}=\{\dot{A} \mid \dot{A}: \check{Y} \rightarrow \mathbf{B}$, where $\left.Y \in[X]^{<\kappa}\right\}$. If $\Gamma \in V[G]$ and $\Gamma \subset[X]^{<\kappa}$, then $\Gamma$ can be represented by a B-name, $\dot{\Gamma}: \mathcal{N} \rightarrow \mathbf{B}$.

THEOREM 5.1. Let $\kappa$ be supercompact. Let $\left(\mathbf{B}_{\alpha}: \alpha \leq \kappa\right)$ be an iterated proper forcing such that for every inaccessible $\nu<\kappa,\left|\mathbf{B}_{\nu}\right|<\kappa$ and $\mathbf{B}_{\kappa} \|-\kappa=\omega_{2}$. Then $\mathbf{B}_{\kappa} \|-$ Axiom R.

ProOF. We may assume that $\mathbf{B}_{\kappa} \subset V_{\kappa} . \mathbf{B}_{\kappa}$ is $\kappa$ cc by a $\Delta$-system argument. Assume that in $V[G], X, \Sigma, \Gamma$ satisfy the hypothesis of Axiom R. Without loss of generality, $X \in V$. Let $\dot{\Sigma}$ and $\dot{\Gamma}$ be $\mathbf{B}_{\kappa}$-names representing $\Sigma$ and $\Gamma$ of the form discussed above. Let $\lambda$ be much bigger than everything mentioned so far, and let $j: V \rightarrow M \supset{ }^{\lambda} V$ be elementary with critical point $\kappa, j \kappa>\lambda$. For every set $S, j^{\prime \prime} S=\{j s: s \in S\} \subset j S$. A careful examination of the form of $\dot{\Sigma}, \dot{\Gamma}$ yields the stronger condition (3) below. (The point is that for $\dot{A} \in \mathcal{N}$, $j(\dot{A})=\{(j x, b):(x, b) \in A\}$. Because $\mathbf{B}_{\kappa} \subset V_{\kappa}, j b=b$ for $b \in B_{\kappa}$. There are no "new" elements of $j(\dot{A})$ because $|\dot{A}|<\kappa$.) It is routine to check that $M \vDash \Psi\left(\kappa, j^{\prime \prime} X, j^{\prime \prime} \dot{\Sigma}, j^{\prime \prime} \dot{\Gamma}, j \kappa, j X, j \dot{\Sigma}, j \dot{\Gamma}\right)$, where $\Psi$ is the conjunction of

(1) $j^{\prime \prime} \dot{\Sigma}$ and $j^{\prime \prime} \dot{\Gamma}$ are $\mathbf{B}_{\kappa}$-names, $j^{\prime \prime} X \in[j X]^{<j \kappa}$.

(2) $\mathbf{B}_{\kappa} \Vdash\left(j^{\prime \prime} \dot{\Sigma}\right.$ is a stationary subset of $\left[j^{\prime \prime} X\right]^{\omega}, j^{\prime \prime} \dot{\Gamma}$ is tight and unbounded in $\left[j^{\prime \prime} X\right]^{<\kappa}$, and $\left.\check{\kappa}=\omega_{2}\right)$.

(3) $\mathbf{B}_{j \kappa} \Vdash j^{\prime \prime} \dot{\Sigma} \subset j \dot{\Sigma}$ and $j^{\prime \prime} \dot{\Gamma} \subset j \dot{\Gamma}$.

Then $M \vDash \exists v_{1} \exists v_{2} \exists v_{3} \exists v_{4} \Psi\left(v_{1}, v_{2}, v_{3}, v_{4}, j \kappa, X, \dot{\Sigma}, j \dot{\Gamma}\right)$. By elementarity

$$
V \vDash \exists v_{1} \exists v_{2} \exists v_{3} \exists v_{4} \Psi\left(v_{1}, v_{2}, v_{3}, v_{4}, \kappa, j X, \dot{\Sigma}, \dot{\Gamma}\right) .
$$

Instantiating, we get $V \vDash \Psi\left(\nu, Y, \dot{\Sigma}^{\prime}, \dot{\Gamma}^{\prime}, \kappa, X, \dot{\Sigma}, \dot{\Gamma}\right)$. Let $G_{\nu}=G \cap \mathbf{B}_{\nu}$. By (1) we can define $\Sigma^{\prime}=\operatorname{val}\left(\dot{\Sigma}^{\prime}, G_{\nu}\right)$ and $\Gamma^{\prime}=\operatorname{val}\left(\dot{\Gamma}^{\prime}, G_{\nu}\right)$. Moreover, by $(2) V\left[G_{\nu}\right] \vDash \Sigma^{\prime}$ is stationary in $[Y]^{\omega}$ and $\Gamma^{\prime}$ is tight and unbounded in $[Y]^{<\nu}$. By properness $\Sigma^{\prime}$ remains stationary in $V[G]$, and $P(Y) \cap \Sigma \supset \Sigma^{\prime}$ is stationary. It remains to show that $Y \in \Gamma$.

Because $V[G] \vDash|Y|=\omega_{1}$, let $Y=\left\{x_{\alpha}: \alpha<\omega_{1}\right\}$. By induction on $\alpha<\omega_{1}$, we will define $A_{\alpha} \in \Gamma^{\prime} \in V\left[G_{\nu}\right]$ so that $\beta<\alpha$ implies $A_{\beta} \cup\left\{x_{\beta}\right\} \subset A_{\alpha}$. Let 
$A_{0} \in \Gamma^{\prime}$ be arbitrary. For $\alpha=\beta+1$, apply $\left(V\left[G_{\nu}\right] \vDash \Gamma^{\prime}\right.$ is unbounded in $\left.[Y]^{<\nu}\right)$ to $A_{\beta} \cup\left\{x_{\beta}\right\}$. For $\alpha$ a limit, $S=\left\{A_{\beta}: \beta<\alpha\right\}$ is probably not in $V\left[G_{\nu}\right]$, but by properness there is $T \in V\left[G_{\nu}\right], T \in\left[\Gamma^{\prime}\right]^{<\nu}$, so that $S \subset T$. Because $V\left[G_{\nu}\right] \vDash \nu$ is $\omega_{2}$, regular, $\bigcup T \in[Y]^{<\nu}$. Apply unbounded to get $A_{\alpha}, \bigcup T \subset A_{\alpha} \in \Gamma^{\prime}$. By construction $\bigcup\left\{A_{\alpha}: \alpha<\omega_{1}\right\}=Y \in \Gamma$, because $\Gamma$ is tight.

6. Digressions. The following three plausible conjectures may summarize and clarify the status of the $S$ 's.

1. $S_{1}, S_{2}$ and $S_{4}$ are equivalent,

2. $S_{3}, S_{5}$ and $S_{6}$ are equivalent; in fact,

3. $S_{3}, S_{5}$ and $S_{6}$ are refutable in ZFC.

RE CONJECTURE 3. In [MS], Milnor and Shelah construct in ZFC an example from Truss of a family, $X$, of $\omega_{2}$ countable sets such that every $Y \in[X]^{<\omega_{2}}$ has a transversal, but $X$ does not. Here, a transversal is a one-to-one choice function.

In the examples of this paper, reflection properties have the form $S\left(P_{1} \& \sim P_{2}\right)$, where $P_{1}$ is hereditary. The contrapositives can be stated without negation. For example, $S_{1}$ : A metric space $X$ is $\sigma$-discrete iff every $Y \in[X]^{<\omega_{2}}$ is $\sigma$-discrete. The "only if" can be added because $\sigma$-discrete is hereditary. Note that $S\left(P_{1} \& \sim P_{2}\right)$ is equivalent to $S\left(P_{1} \& \sim\right.$ hereditarily $\left.P_{2}\right)$ because a subset of a small set is small.

We stated the $S$ 's as reflection properties and proved some of them by reflection arguments. The contrapositives can be proven by integrating winning tactics $t_{Y}$, $Y \in[X]^{<\omega_{2}}$, into a winning strategy for $X$ (see Theorem 2.2(b), (b')) using a homomorphism $h: P\left([X]^{<\omega_{2}}\right) \rightarrow \mathbf{B}$, where $\mathbf{B}$ is a proper complete Boolean algebra. For specific Boolean algebras, a strongly compact cardinal, rather than a supercompact cardinal, suffices $\left[\mathbf{F}_{\mathbf{2}}\right]$.

A timely letter from Peg Daniels reminded me of another reflection that can be proven in ZFC.

THEOREM 6.1. Let $X$ be a $T_{1}$ space with a point-countable base such that $|X|=\lambda$, singular, and every $Y \in[X]^{<\omega_{2}}$ is left separated. Then $X$ is left separated.

PROOF. We follow the proof in $\left[\mathbf{F}_{4}\right]$ of the analogous results for locally small spaces and coloring numbers. Let $\left(\lambda_{\alpha}\right)_{\alpha<\operatorname{cf} \lambda}$ be a continuous, increasing sequence of cardinals cofinal in $\lambda$ with $\lambda_{0} \geq \mathrm{cf} \lambda$. By induction on $\beta \leq \omega_{1}$, we will define continuous increasing sequences $\mathcal{Y}_{\beta}=\left\{Y_{\beta \alpha}: \alpha<\operatorname{cf} \lambda\right\}$ such that

$$
\left|Y_{\beta \alpha}\right|=\lambda_{\alpha} \quad \text { and } \quad \bigcup \mathcal{U}_{\beta}=X \text {. }
$$

Let $\mathcal{Y}_{0}$ be any sequence satisfying $(*)$. If $\beta=\gamma+1$, by the proof of Lemma 4.1 we can enumerate $\bar{Y}_{\gamma \alpha}$ as $\left\{x_{\alpha \xi}^{\gamma}: \xi<\lambda_{\alpha}\right\}$ for each $\alpha$. Set $Y_{\beta \alpha}=\left\{x_{\delta \xi}^{\gamma}\right\}: \xi<\lambda_{\alpha}$, $\delta<\operatorname{cf} \lambda\} .\left|Y_{\beta \alpha}\right|=\mathrm{cf} \lambda \cdot \lambda_{\alpha}=\lambda_{\alpha}$.

If $\beta$ is a limit, set $Y_{\beta \alpha}=\bigcup\left\{Y_{\gamma \alpha}: \gamma<\beta\right\}$. By countable tightness, $y_{\omega_{1}}$ is a continuous, increasing sequence of closed subsets of $X$. Thus we can piece together well orderings of $Y_{\omega_{1} \alpha+1}-Y_{\omega_{1} \alpha}$ to left separate $X$ (as in $2.2\left(\mathrm{c}^{\prime}\right) \rightarrow(\mathrm{a})$ ).

It is clear that the "right" generalization of countability in Euclidean space to arbitrary metrizable spaces is $\sigma$-discreteness. Theorem 2.2 suggests that the "right" further generalization is left separation in spaces with point countable base. In a similar context, Balogh and Junnila $[\mathbf{B J}]$ generalized a theorem about $\sigma$ discreteness in metric spaces to $\sigma$-left separation in $T_{1}$ spaces of character $\leq \mathfrak{c}$. They were able to achieve $\sigma$-relatively discrete only with additional hypotheses. 


\section{REFERENCES}

[Au] C. Aull, Topological spaces with a $\sigma$-point finite base, Proc. Amer. Math. Soc. 29 (1971), 411-416.

[BJ] Z. Balogh and H. Junnila, Totally analytic spaces under $V=L$, Proc. Amer. Math. Soc. 87 (1983), 514-527.

[Ba $]$ J. Baumgartner, Applications of the proper forcing axiom, Handbook of Set Theoretic Topology (K. Kunen and J. Vaughan, eds.), North-Holland, Amsterdam, 1984.

[Ba2 $\ldots$, Iterated forcing, Surveys in Set Theory (A. Mathias, ed.), Cambridge Univ. Press, 1983.

[BMR] J. Baumgartner, J. Malitz and W. Reinhart, Embedding trees in the rationals, Proc. Nat. Acad. Sci. U.S.A. 67 (1970), 1748-1753.

[DS] K. Devlin and S. Shelah, A weak version of $\diamond$ which follows from $2^{\aleph_{0}}<2^{\aleph_{1}}$, Israel J. Math. 29 (1978), 239-247.

[vD] E. van Douwen, The integers and topology, Handbook of Set Theoretic Topology (K. Kunen and J. Vaughan, eds.), North-Holland, Amsterdam, 1984.

[EL] R. Engelking and D. Lutzer, Paracompactness in ordered spaces, Fund. Math. 94 (1977), 49-58.

[F. W. Fleissner, An axiom of nonseparable Borel theory, Trans. Amer. Math. Soc. 251 (1979), 309-328.

$\left[\mathbf{F}_{\mathbf{2}}\right]$ - Homomorphism axioms and lynxes, Contemp. Math. 31 (1984), 79-97.

$\left[\mathbf{F}_{3}\right]$, Separation properties in Moore spaces, Fund. Math. 98 (1978), 279-286.

$\left[\mathbf{F}_{4}\right] \_$, On $\lambda$-collectionwise Hausdorff spaces, Topology Proc. 2 (1978), 445-458.

[Fo] G. Fodor, Proof of a conjecture of Erdös, Acta. Sci. Math. Szeged 14 (1952), 219-227.

[Fr] D. Fremlin, Measure-additive coverings and measurable selectors, Dissertationes Math. (to appear).

[Ha] R. Hansell, Point-countable Souslin-additive families and $\sigma$-discrete reduction, Proc. Fifth Prague Topology Sympos. (J. Novak, ed.), 1983.

[KM] A. Kanamori and M. Magidor, The evolution of large cardinal axioms in set theory, Lecture Notes in Math., vol. 669, Springer-Verlag, Berlin and New York, 1978. pp. 99-275.

[Kue] D. Kueker, Countable approximations and Lowenheim-Skolem theorems, Ann. Math. Logic 11 (1977), 57-103.

[Kun] K. Kunen, Set theory: An introduction to independence proofs, North-Holland, Amsterdam, 1980.

[MS] E. Milner and S. Shelah, Some theorems on transversals, Infinite and Finite Sets (A. Hajnal, T. Rado and V. Sós, eds.), North-Holland, Amsterdam, 1975, pp. 1115-1126.

[Mi] A. Mišcenko, Spaces with point countable base, Dokl. Akad. Nauk SSSR 144 (1962), 985988=Soviet Math. Dokl. 3 (1962), 855-858.

[Po] R. Pol, Note on decompositions of metric spaces. II, Fund. Math. 100 (1978), 129-143.

[Sh 1 S. Shelah, Proper forcing, Lecture Notes in Math., vol. 940, Springer-Verlag, Berlin and New York, 1982.

[Sh $\left.\mathrm{Sh}_{2}\right]$, Remarks on $\lambda$-collectionwise Hausdorff spaces, Topology Proc. 2 (1977), 583-592.

[ $\left.\mathbf{T}_{\mathbf{1}}\right]$ F. Tall, Normality versus collectionwise normality, Handbook of Set Theoretic Topology (K. Kunen and J. Vaughan, eds.), North-Holland, Amsterdam, 1984.

$\left[\mathbf{T}_{\mathbf{2}}\right]$, Some reflections on collectionwise normality (to appear).

[To 1 S. Todorcevic, Some consequences of MA+ KH, Topology Appl. 12 (1981), 187-202.

$\left[\mathbf{T o}_{2}\right]-$, Trees and linearly ordered sets, Handbook of Set Theoretic Topology (K. Kunen and J. Vaughan, eds.), North-Holland, Amsterdam, 1984.

[Wi] N. Williams, Combinatorial set theory, North-Holland, Amsterdam, 1977. 78712

Department of Mathematics, University of TeXas at Austin, Austin, Texas

Current address: Department of Mathematics, University of Pittsburgh, Pittsburgh, Pennsylvania 15260 